05

\title{
Влияние границ зерен на электропроводность медных сплавов
}

\author{
(C) Р.Г. Чембарисова \\ Уфимский государственный авиационный технический университет, \\ 450008 Уфра, Россия \\ e-mail: chroza@yandex.ru
}

Поступило в Редакцию 1 июля 2019 г.

В окончательной редакции 1 июля 2019 г.

Принято к публикации 21октября 2019 г.

\begin{abstract}
Методами аналитического моделирования проведен анализ выявленного экспериментально отсутствия чувствительности удельного сопротивления сплавов $\mathrm{Cu}-\mathrm{Cr}, \mathrm{Cu}-\mathrm{Cr}-\mathrm{Zr}$ к структурным изменениям, наблюдаемым в образцах, подвергнутых интенсивной пластической деформации. На примере сплава $\mathrm{Cu}-1.8$ wt.\% $\mathrm{Cr}-0.8$ wt.\% $\mathrm{Zr}$ показано, что увеличение вклада в удельное сопротивление границ зерен и выделившихся мелких частиц вторичных фаз после равноканального углового прессования компенсируется преимущественно уменьшением вклада в удельное сопротивление растворенных в матрице атомов легирующих элементов.
\end{abstract}

Ключевые слова: медные сплавы, удельное сопротивление, ультрамелкозернистое состояние, границы зерен, частицы вторичных фаз.

DOI: $10.21883 /$ JTF.2020.04.49087.250-19

\section{Введение}

Ультрамелкозернистые (УМЗ) и нанокристаллические (НК) материалы демонстрируют уникальные физические свойства, отличные от тех, которые характерны для их крупнокристаллических (КК) аналогов. Эти свойства часто вытекают из того факта, что объемная доля границ зерен (ГЗ) в таких материалах на несколько порядков выше, чем в тех же материалах в их КК состоянии. В УМЗ и НК материалах ГЗ являются в основном высокоугловыми, имеют более высокое значение избыточного объема и характеризуются высоким уровнем внутренних напряжений.

Известно, что влияние ГЗ на удельное сопротивление обычных поликристаллических материалов с размером зерна, равным десяткам и сотням микрон, незначительно благодаря малой длине ГЗ в структуре таких материалов. Однако в УМЗ и НК материалах протяженность ГЗ значительно возрастает. В НК материалах размеры зерен могут стать даже сопоставимыми с длиной свободного пробега электронов проводимости в образце.

Для получения объемных УМЗ материалов применяют методы интенсивной пластической деформации (ИПД): кручение под высоким давлением, равноканальное угловое прессование (РКУП) и т.д. УМЗ материалы, полученные в процессе ИПД и последующего рекристаллизационного отжига, свободны от пор. Поэтому изменения их удельного сопротивления обусловлены главным образом изменением протяженности ГЗ. Авторы работы [1] подвергли образцы поликристаллической меди чистотой 99.98\% кручению под высоким давлением и последующему рекристаллизационному отжигу в вакууме в интервале температур от 50 до $900^{\circ} \mathrm{C}$, что позволило изучить зависимость удельного сопротивле- ния от среднего размера зерен в широком интервале изменения размера - от субмикронных до десятков микрон. Было показано, что пока размеры зерен больше $10 \mu \mathrm{m}$, удельное сопротивление образцов, измеренное при температуре $77 \mathrm{~K}$, изменяется слабо. С дальнейшим уменьшением их размеров рост удельного сопротивления становился более заметным, а в субмикронной области наблюдалось его резкое увеличение. Аналогичная тенденция была выявлена в УМЗ никеле чистотой 99.99\% с размерами фрагментов во фрагментированной структуре, равными $0.1-0.2 \mu \mathrm{m}$ и низкой плотностью дислокаций во фрагментах [2]. Углы разориентации были высокими. В интервале температур отжига от комнатной до $175^{\circ} \mathrm{C}$ размеры зерен практически не изменялись. Считалось, что в интервале температур от 175 до $300^{\circ} \mathrm{C}$ удельное сопротивление является функцией среднего размера зерен. Соответственно с увеличением температуры отжига (размера зерен) наблюдалось уменьшение удельного сопротивления образца.

Увеличение содержания легирующего элемента приводит к формированию в результате ИПД меньшего размера зерна и повышению прочностных свойств материала [3,4]. Увеличение прочности металлических материалов за счет введения в структуру различных препятствий движению дислокаций сопровождается снижением их электропроводности $[1,2,5,6]$. В то же время в работах $[7,8]$ показано, что увеличение электропроводности сплавов может быть достигнуто в результате выделения растворенных атомов в частицы вторичной фазы в процессе старения. Например, авторы работы [8] подвергли сплав $\mathrm{Cu}-0.5 \mathrm{wt} . \% \mathrm{Cr}$ холодной прокатке после 4 проходов РКУП и последующему старению в течение $1 \mathrm{~h}$ при $450^{\circ} \mathrm{C}$, что привело к увеличению прочности при растяжении до $554 \mathrm{MPa}$, электропроводности 
до 84\% IACS. После 4 проходов РКУП прочность была равна $460 \mathrm{MPa,} \mathrm{электропроводность} \mathrm{-} \approx 35 \%$ IACS (International Annealed Copper Standart). Возросшая прочность образцов сплава в данном случае объяснялась измельчением зерна и выделением частиц вторичной фазы, увеличение электропроводности - выделением легирующих атомов из матрицы в частицы вторичной фазы. В то же время оказалось, что электропроводность образцов практически не зависит от числа проходов РКУП. Таким образом, удельное сопротивление сплава $\mathrm{Cu}-0.5 \mathrm{wt.} \% \mathrm{Cr}$ не зависело от размера зерна и сформировавшихся в процессе ИПД дефектов кристаллической решетки. Отсутствие зависимости электрической проводимости от размера зерна (протяженности ГЗ) и внесенных в результате ИПД дефектов была выявлена и в сплавах системы $\mathrm{Cu}-\mathrm{Cr}-\mathrm{Zr}$ [4]. Исходное состояние этих сплавов было сформировано в результате высокотемпературной термообработки при температуре $1000^{\circ} \mathrm{C}$ в течение $0.5 \mathrm{~h}$ с последующей закалкой в $5 \%$ растворе $\mathrm{NaCl}$. Полученные образцы подвергли РКУП при комнатной температуре. Оказалось, что удельные сопротивления сплавов, подвергнутых РКУП, совпадают в пределах погрешностей с удельными сопротивлениями в их исходных состояниях. Целью настоящей работы является анализ на основе аналитических моделей влияния элементов микроструктуры на электропроводность сплавов системы $\mathrm{Cu}-\mathrm{Cr}-\mathrm{Zr}$ (на примере сплава $\mathrm{Cu}-1.8$ wt.\% $\mathrm{Cr}-0.8$ wt.\% $\mathrm{Zr}$ ) в исходных (SS) состояниях и в состояниях, сформировавшихся в процессе 4 проходов РКУП (4ЕСАР), что позволит объяснить отсутствие выраженной зависимости их удельного сопротивления от протяженности границ зерен и сформировавшихся в результате ИПД дефектов кристаллической структуры.

\section{1. Электропроводимость металлических материалов}

Удельное сопротивление металлических материалов определяется рассеянием электронов на различных дефектах кристаллической решетки. Электроны могут рассеиваться и на атомах в узлах кристаллической решетки. При этом сечение рассеяния электронов определяется зависящей от температуры амплитудой колебаний атомов. При высоких температурах $T, T \gg \Theta$, где $\Theta$ - температура Дебая, удельное сопротивление растет линейно с увеличением температуры. В промежуточной области температур удельное сопротивление пропорционально температуре в пятой степени, $\rho \sim T^{5}$ (закон Блоха-Грюнайзена) [6,9]. С приближением к гелиевой температуре, равной $4.2 \mathrm{~K}$, удельное сопротивление стремится к некоторой постоянной величине. Рассеяние электронов на дефектах кристаллического строения материала не зависит от температуры. При низких температурах удельное сопротивление металлических материалов определяется рассеянием электронов на дефектах кристаллической решетки, поскольку вклад тепловых колебаний атомов решетки стремится к нулю.

\section{1. Вклад дислокаций в удельное сопротивление металлов}

Для вычисления удельного сопротивления, обусловленного наличием в материале дислокаций, можно воспользоваться методом, описанным в работах $[5,10]$. Было принято, что рассеяние электронов происходит на ядре дислокации, которое представлялось в виде отрицательно заряженного полого цилиндра. При этом использовалась теория возмущений, и рассматривалось экранирование в приближении Томаса-Ферми. Аппроксимация цилиндрического ядра прямоугольным параллелепипедом значительно упрощает процедуру интегрирования при решении задачи. Поэтому область взаимодействия электрона с ядром дислокации была представлена в виде прямоугольного параллелепипеда, объем которого равен $L l^{2}$. Размеры области действия рассеивающего потенциала определялись исходя из величины дилатации решетки в области ядра дислокации. В работе [11] было показано, что изменение объема кристалла $\Delta V$ на единицу длины дислокации, вызванное дислокациями, складывается из неупругой дилатации в области ядра дислокации $\Delta V_{c}=2 b_{\mathrm{B}}^{2}\left(b_{\mathrm{B}}-\right.$ величина вектора Бюргерса) и упругого изменения объема кристалла $\Delta V_{s}$ за исключением области ядра $\Delta V_{s}=-b_{\mathrm{B}}^{2}$. Соответственно $\Delta V=b_{\mathrm{B}}^{2}$. Однако в сильно деформированной нелинейной упруго изотропной среде $\Delta V>b_{\mathrm{B}}^{2}$, возможно, даже $\Delta V \geq 2 b_{\mathrm{B}}^{2}$. Сечение рассеяния электронов определяется величиной избыточного заряда, вносимого дефектом в решетке. Поэтому геометрическая форма пустот в ГЗ не существенна.

Усредненная вероятность рассеяния в единицу времени для электрона с волновым числом $k$ на $N_{d i s l}$ дислокациях была представлена формулой [10]:

$$
\left\langle P_{N}\right\rangle=\frac{1}{\tau_{k}}=\frac{k^{2} \hbar l^{2} L}{9 m^{*} \Omega} \rho_{t o t} L^{2}\left[1-\frac{\sin 2 k l}{2 k l}\right],
$$

которая позволяет вычислить удельное сопротивление $\rho_{\text {disl }}$, обусловленное рассеянием на дислокациях, вида $[5]$ :

$$
\frac{\rho_{\text {disl }}}{\rho_{\text {tot }}}=\frac{\hbar k^{2} l^{2}}{9 n_{e} e^{2}}\left(1-\frac{\sin 2 k l}{2 k l}\right),
$$

где $\tau_{k}$ - время релаксации, $\rho_{t o t}-$ плотность дислокаций, $L-$ длина дислокации, $\Omega-$ объем образца, $l^{2}-$ сечение ядра дислокации, $k=2 \pi / \lambda-$ волновое число, $\lambda=h / m^{*} u_{\mathrm{F}}$ - длина волны электрона, $h(\hbar=h / 2 \pi)-$ постоянная Планка, $m^{*}$ - эффективная масса электрона, $n_{e}$ - объемная плотность электронов, $e-$ величина заряда электрона, $u_{\mathrm{F}}-$ скорость Ферми. Размеры области рассеяния электрона были приняты равными $l \approx 1.55 b_{\text {В }}$ в соответствии с данными работ $[11,12]$. 


\section{2. Вклад границ зерен в удельное сопротивление металлических материалов}

В УМЗ и НК материалах плотность ГЗ на несколько порядков выше, чем в их КК аналогах. Поэтому влияние ГЗ на электропроводящие свойства материала возрастает. В работе [13] предложена модель границ зерен, позволяющая в рамках единого формализма рассматривать ГЗ с любой разориентировкой. Она была успешно применена для вычисления вклада ГЗ в удельное сопротивление объемных одновалентных, поливалентных и переходных металлов [14]. Предполагалось, что рассеяние электронов происходит только в области ядер дислокаций, образующих границу, так как вклад дислокационных полей упругих напряжений в удельное сопротивление на два порядка ниже [15]. Считалось, что дислокации в границе не взаимодействуют друг с другом.

Структура малоугловой границы достаточно хорошо изучена [13]. Простую симметричную границу наклона в кубической решетке можно представить одним набором краевых дислокаций, которые отстоят друг от друга на расстояние

$$
h=\frac{b_{\mathrm{B}}}{2 \sin (\Theta / 2)} .
$$

Для описания границ общего вида необходимы три независимых набора дислокаций с некомпланарными векторами Бюргерса. Увеличение плотности дислокаций сопровождается увеличением угла разориентировки между соседними зернами. Как было показано в работе [12], при углах, превышающих 20-25, понятие отдельных дислокаций теряет смысл. Общим для множества существующих моделей структуры ГЗ с большими углами разориентировки является отражение того наблюдаемого экспериментально факта, что высокоугловые границы являются более рыхлыми и пористыми по сравнению с малоугловыми границами.

Дислокации - линейные дефекты кристаллической решетки, на которых происходит рассеяние электронов. В работе [13] представлена модель изменения строения ядер дислокаций, образующих симметричную границу наклона. В малоугловой области граница представляется дислокациями с векторами Бюргерса $b_{\mathrm{B}}$ решеточных дислокаций. В случае ГЦК решетки $b_{\mathrm{B}}=a \sqrt{2} / 2$, где $a$ - период решетки.

С ростом плотности дислокаций, когда расстояние между дислокациями оказывается меньше некоторого критического значения $h_{c}$, дислокации начинают объединяться в сверхдислокации с вектором Бюргерса $b$. Расчеты, выполненные в работах $[16,17]$, показали, что процесс слияния дислокаций возможен при расстояниях между ними $h=(5-10) b_{\mathrm{B}}$. С началом этого процесса можно говорить об увеличении среднего вектора Бюргерса $b$ сверхдислокации. Считается, что величина вектора Бюргерса изменяется с ростом угла разориентации $\Theta$ по закону $b=2 h_{c} \sin (\Theta / 2)$. При некотором значении угла $\Theta=\Theta^{*}$ граница становится высокоугловой [18] в соответствии с данными Гляйтера [12] о том, что в высокоугловой области отмечается уширение ядер зернограничных дислокаций. При $\Theta>\Theta^{*}$ граница считается высокоугловой, состоящей из цилиндрических отрицательно заряженных пор. Ее структура и параметры ядер линейных дефектов не зависят от угла разориентации, что подтверждается независимостью энергии границ $E_{G}(\Theta)$ от угла $\Theta$ в высокоугловой области, если в ней отсутствуют специальные разориентировки [13]. Специальные границы с высокой плотностью узлов совпадения представляют собой срелаксированные границы, характеризующиеся спадом энергии в зависимости $E_{G}(\Theta)$. Структура их аналогична малоугловым, т. е. носит дислокационный характер $[12,19]$.

В работе [14] показано, что в кубических структурах при малых углах разориентации $\left(\Theta<\Theta_{c}\right)$ величина вектора Бюргерса равна $b=b_{\mathrm{B}}$. В переходной области $\Theta_{c} \leq \Theta \leq \Theta^{*}, b=2 h_{c} \sin (\Theta / 2), h_{c}=7 b_{\mathrm{B}}$. В области высоких углов разориентации $\Theta^{*}<\Theta \leq \Theta_{s} / 2$, $b=2 h_{c} \sin \left(\Theta^{*} / 2\right)$. $\Theta_{c}$ и $\Theta^{*}-$ углы, разделяющие эти области. Для меди $\Theta_{c}=8^{\circ}, \Theta^{*}=16^{\circ} . \Theta_{s} / 2-$ угол максимальной разориентировки. Для кубических структур $\Theta_{s}=\pi / 2$.

Рассмотрим простую симметричную границу в кубической решетке. Зная удельное сопротивление линейных дефектов, составляющих границу зерна, на единицу плотности дислокаций в ГЗ $\rho_{\text {disl }} / \rho_{w}$ и расстояние между ними $h$, можно вычислить удельное сопротивление на единицу площади ГЗ в единице объема поликристаллического материала $\rho_{g b} / N_{g b}[14]$ :

$$
\frac{g_{g b}}{N_{g b}}=\frac{\rho_{\text {disl }}}{\rho_{w}} \frac{1}{h}
$$

где $\rho_{w}-$ плотность дислокаций в ГЗ, $\rho_{g b}-$ вклад ГЗ в удельное сопротивление материала, $N_{g b}=S / \Omega, S-$ площадь границ в объеме $\Omega$. Для кубических структур с учетом значения величины $h(2)$ формулу (3) можно записать в виде

$$
\frac{g_{g b}}{N_{g b}}=\frac{\rho_{d i s l}}{\rho_{w}} \frac{2 \sin (\Theta / 2)}{b} .
$$

Удельное сопротивление единицы плотности дислокаций (1) в ГЗ КК материалов примем равным $2.540 \cdot 10^{-25} \Omega \cdot \mathrm{m}^{3}$, согласно известным экспериментальным данным о среднем по всем углам разориентации удельном сопротивлении ГЗ $\left\langle\rho_{g b} / N_{g b}\right\rangle$ на единицу их плотности в КК материалах, приведенным в работе [14]. При этом среднее по всем углам разориентации удельное сопротивление ГЗ на единицу их плотности вычислялось согласно формуле

$$
\begin{aligned}
\left\langle\frac{\rho_{g b}}{N_{g b}}\right\rangle & =\frac{2}{\Theta_{s}} \int_{0}^{\Theta_{s} / 2} \frac{\rho_{\text {disl }}}{\rho_{w}} \frac{2 \sin (\Theta / 2)}{b} d \Theta \\
& =2.096 \cdot 10^{-16} \Omega \cdot \mathrm{m}^{2} .
\end{aligned}
$$


Среднее значение удельного сопротивления на единицу площади ГЗ в единице объема с углами разориентации $\Theta<\Theta_{c}$ будет равно

$$
\begin{aligned}
\left\langle\frac{\rho_{g b}}{N_{g b}}\right\rangle & =\frac{1}{\Theta_{c}} \int_{0}^{\Theta_{c}} \frac{\rho_{d i s l}}{\rho_{w}} \frac{2 \sin (\Theta / 2)}{b} d \Theta \\
& =0.693 \cdot 10^{-16} \Omega \cdot \mathrm{m}^{2} .
\end{aligned}
$$

Среднее значение удельного сопротивления на единицу площади ГЗ в единице объема с углами разориентации $\Theta<\Theta^{*}$ составит

$$
\begin{aligned}
\left\langle\frac{\rho_{g b}^{l}}{N_{g b}}\right\rangle & =\frac{1}{\Theta^{*}} \int_{0}^{\Theta^{*}} \frac{\rho_{d i s l}}{\rho_{w}} \frac{2 \sin (\Theta / 2)}{b} d \Theta \\
& =1.054 \cdot 10^{-16} \Omega \cdot \mathrm{m}^{2} .
\end{aligned}
$$

В области высоких углов разориентации среднее значение удельного сопротивления на единицу площади ГЗ в единице объема примет значение

$$
\begin{aligned}
\left\langle\frac{\rho_{g b}^{h}}{N_{g b}}\right\rangle & =\frac{4}{\Theta_{s}-2 \Theta^{*}} \int_{\Theta^{*} / 2}^{\Theta_{s} / 4} \frac{\rho_{\text {disl }}}{\rho_{w}} \frac{2 \sin Z}{b} d Z \\
& =2.675 \cdot 10^{-16} \Omega \cdot \mathrm{m}^{2} .
\end{aligned}
$$

Имеющееся экспериментальное значение величины $\left\langle\rho_{g b}^{h} / N_{g b}\right\rangle$ для УМЗ $\mathrm{Cu}[20]$, рассчитанное из графика при значении размера зерна $d=160 \mathrm{~nm}$, согласуется с полученным, согласно формуле (5), значением, равным $5.6 \cdot 10^{-16} \Omega \cdot \mathrm{m}^{2}$, соответствующим значению удельного сопротивления единицы плотности дислокаций (1) в ГЗ УМЗ материалов, равному $5.32 \cdot 10^{-25} \Omega \cdot \mathrm{m}^{3}$.

\section{3. Вклад растворенных атомов и вакансий в удельное сопротивление металлических материалов}

Типичными дефектами кристаллической решетки являются вакансии и чужеродные атомы. Связанный с точечными дефектами потенциал был выбран равным $U=n_{e} / g(E)=(2 / 3) E_{\mathrm{F}}$, где $g(E)$ - плотность квантовых состояний в металле, $E_{\mathrm{F}}-$ энергия Ферми [10]. Взаимодействие электрона с потенциалом точечного дефекта и вызовет его рассеяние. При этом было принято во внимание, что мы имеем дело с вырожденным Ферми-газом электронов. Соответственно процессы упругого рассеяния возможны только для электронов, которые в импульсном пространстве находятся вблизи поверхности Ферми. Во внутренних областях сферы Ферми процессы рассеяния запрещены согласно принципу Паули. Соответственно длина волны рассеиваемых электронов оказывается одной и той же при всех температурах $T$.
Для вычисления вклада растворенных в матрице атомов и вакансий в удельное сопротивление исследуемого материала можно воспользоваться методом, использованным для определения удельного сопротивления, обусловленного дислокациями. При этом область взаимодействия электрона с точечным дефектом представляется в виде кубика, объем которого равен $l^{3}$. Вклад в удельное сопротивление растворенных атомов (вакансий) тогда может быть оценен по формуле, представленной в работе [10]:

$$
\rho_{a}=\frac{k^{3} \hbar l^{4} n_{a} C_{s a}}{9 e^{2} \pi n_{e}}\left[1-\frac{\sin 2 k l}{2 k l}\right],
$$

где $C_{s a}$ - атомная доля растворенных атомов (вакансий $), n_{a}$ - объемная концентрация атомов матрицы, $l-$ размеры области взаимодействия электрона с чужеродным атомом (вакансией) [10].

Массовая доля $W_{s a}$ растворившихся в матрице атомов компонента $a$ равна

$$
W_{s a}=\frac{m_{s a}}{m_{a}+m_{b}+m_{m}},
$$

где $m_{s a}$ - масса растворившегося в матрице вещества $a$; $m_{a}, m_{b}$ - массовое содержание веществ $a$ и $b$ в сплаве, $m_{m}$ - масса матрицы. Из определения массовой доли атомов вещества $a$ в растворе (7) следует, что

$$
n_{s a}=\frac{N_{s a}}{N_{m}}=W_{s a}\left(n_{a} \frac{\mu_{a}}{\mu_{m}}+n_{b} \frac{\mu_{b}}{\mu_{m}}+1\right) \frac{\mu_{m}}{\mu_{a}},
$$

где $N_{s a}$ - число растворившихся атомов компоненты $a$; $N_{m}$ - число атомов матрицы; $\mu_{a}, \mu_{m}$ - относительные атомные массы вещества $a$ и матрицы соответственно

$$
n_{a}=\frac{W_{a}}{1-W_{a}-W_{b}} \frac{\mu_{m}}{\mu_{a}}, n_{b}=\frac{W_{b}}{1-W_{a}-W_{b}} \frac{\mu_{m}}{\mu_{b}},
$$

где $W_{a}, W_{b}$ - массовые доли веществ $a$ и $b$ в сплаве. Доля $C_{s a}$ растворившихся в матрице атомов вещества $a$ при этом равна

$$
C_{s a}=\frac{N_{s a}}{N_{a}+N_{b}+N_{m}}=\frac{n_{s a}}{n_{a}+n_{b}+1},
$$

где $N_{a}, N_{b}$ - число атомов веществ $a$ и $b$ соответственно. Аналогично определяется доля $C_{s b}$ растворившихся в матрице атомов компонента $b$.

\section{4. Вклад частиц вторичной фазы в удельное сопротивление металла}

Вклад частиц вторичной фазы в удельное сопротивление, согласно приведенной в работе [21] формуле, можно представить в виде

$$
\rho_{\text {seca }} \approx \frac{m_{e} u_{\mathrm{F}} f_{\text {seca }}}{n_{a} e^{2}} \frac{\pi}{V_{p a}}(R+r),^{2}
$$

где $R$ - радиус области взаимодействия электрона с частицей, $r-$ длина экранирования, 
Таблица 1. Параметры микроструктуры сплава $\mathrm{Cu}-1.8 \mathrm{Cr}-0.8 \mathrm{Zr}$ в исходном SS состоянии и в состоянии 4ЕCAP [4]

\begin{tabular}{c|c|c|c|c|c}
\hline Состояние & $D_{X R D}, \mathrm{~nm}$ & $\left\langle\varepsilon^{2}\right\rangle^{1 / 2}, \%$ & $a, \AA$ & $d_{a v}, \mathrm{~nm}$ & $d, \mathrm{~nm}$ \\
\hline SS & - & - & $3.62167 \pm 0.00005$ & $(2.6 \pm 0.2) \cdot 10^{3}$ & $(110 \pm 4) \cdot 10^{3}$ \\
4ECAP & $34 \pm 4$ & $0.20 \pm 0.02$ & $3.62025 \pm 0.00002$ & $(1.9 \pm 0.1) \cdot 10^{3}$ & $155 \pm 10$
\end{tabular}

$r \approx 3 \cdot 0.554 \cdot 10^{-10} \mathrm{~m}, V_{p a}$ - объем частицы вторичной фазы $a$.

Из определения массовой доли $W_{\text {seca }}$ выделившихся в частицы вторичной фазы атомов компоненты $a$ :

$$
W_{\text {seca }}=\frac{m_{\text {seca }}}{m_{a}+m_{b}+m_{m}},
$$

где $m_{\text {seca }}$ - масса выделившихся в частицы вторичной фазы атомов компоненты $a$, следует, что

$$
n_{\text {seca }}=\frac{N_{\text {seca }}}{N_{m}}=W_{\text {seca }}\left(n_{a} \frac{\mu_{a}}{\mu_{m}}+n_{b} \frac{\mu_{b}}{\mu_{m}}+1\right) \frac{\mu_{m}}{\mu_{a}},
$$

где $N_{\text {seca }}$ - число выделившихся в частицы атомов компоненты $a$. При этом их атомная доля равна

$$
C_{\text {seca }}=\frac{N_{\text {seca }}}{N_{a}+N_{b}+N_{m}}=\frac{n_{\text {seca }}}{n_{a}+n_{b}+1} .
$$

В то же время формулу (9) можно представить в виде

$$
C_{\text {seca }}=\frac{N_{p a} N_{p}}{N_{a}+N_{b}+N_{m}},
$$

где $n_{a}=N_{a} / N_{m}, n_{b}=N_{b} / N_{m} ; N_{p a}$ - число атомов компоненты $a$ в одной частице; $N_{p}$ - число частиц. Тогда

$$
N_{p}=\frac{C_{\text {seca }}\left(N_{a}+N_{b}+N_{m}\right)}{N_{p a}} .
$$

Объемная доля частиц вторичной фазы $f_{\text {seca }}$ равна

$$
f_{\text {seca }}=\frac{V_{p a} N_{p}}{V_{s}},
$$

где $V_{s}$ - объем образца. Аналогично определяется объемная доля $f_{\text {secb }}$ частиц вторичной фазы, содержащих атомы вещества $b$.

\section{2. Удельное сопротивление сплавов системы $\mathrm{Cu}-\mathrm{Cr}-\mathrm{Zr}$ в исходном состоянии}

Согласно данным, представленным в работе [4], исходное состояние сплавов системы $\mathrm{Cu}-\mathrm{Cr}-\mathrm{Zr}$ было сформировано в результате высокотемпературной термообработки при температуре $1000^{\circ} \mathrm{C}$ в течение $0.5 \mathrm{~h}$ с последующей закалкой в 5\% растворе $\mathrm{NaCl}$. Растворимость $\mathrm{Cr}$ в $\mathrm{Cu}$ при $1000^{\circ} \mathrm{C}$ составляет 0.4 wt.\% (0.488 at.\%), растворимость $\mathrm{Zr}$ в меди - 0.15 wt.\% (0.104 at.\%) [22]. Поскольку содержание легирующих элементов превышает их растворимость в $\mathrm{Cu}$ при температуре закалки, в структуре сплавов появляются частицы нерастворившихся фаз.

В литом состоянии структура сплавов на основе меди $\mathrm{Cu}-\mathrm{Cr}, \mathrm{Cu}-\mathrm{Zr}, \mathrm{Cu}-\mathrm{Cr}-\mathrm{Zr}$ состоит из дендритов твердого раствора с распределенными по границам дендритов частицами фаз в соответствии с диаграммой состояния [23]. В сплавах, содержащих $\mathrm{Cr}$, из твердого раствора выделяются частицы $\mathrm{Cr}$ [24] со средним размером $\sim 1.5 \mu \mathrm{m}$. В сплавах, содержащих $\mathrm{Zr}$, присутствуют более крупные $(3.5 \mu \mathrm{m})$ частицы интерметаллидного соединения $\mathrm{Cu}_{5} \mathrm{Zr}$ со сложной кубической решеткой типа $\mathrm{AuBe}_{5}$ [25]. Повышение плотности дислокаций и концентрации вакансий облегчает выделение частиц $\mathrm{Cu}_{5} \mathrm{Zr}$. Морфология этих частиц подобна их морфологии в двойных сплавах. Фаза $\mathrm{Cu}_{5} \mathrm{Zr}$ выделяется гетерогенно на дислокациях. Растворимость меди в хроме в твердом состоянии ничтожно мала. Поэтому $\beta$-фаза - твердый раствор на основе $\mathrm{Cr}$, которая существует наряду с твердым раствором на основе меди $(\mathrm{Cu})$ в системе $\mathrm{Cu}-\mathrm{Cr}$, является практически чистым хромом (Cr) [23]. В сплавах, содержащих $\mathrm{Cr}$ и $\mathrm{Zr}$, присутствуют частицы на основе $\mathrm{Cr}$ и частицы интерметаллидного соединения $\mathrm{Cu}_{5} \mathrm{Zr}$. Последующая горячая ковка и закалка приводят к формированию более равномерной структуры со средним размером зерна $150-200 \mu \mathrm{m}$ и к частичному растворению вторичных фаз [23].

Проведем анализ влияния элементов микроструктуры на электропроводность сплавов системы $\mathrm{Cu}-\mathrm{Cr}-\mathrm{Zr}$ на примере сплава $\mathrm{Cu}-1.8$ wt. $\% \mathrm{Cr}-0.8$ wt.\% $\mathrm{Zr}$ методом аналитического моделирования.

Согласно экспериментальным данным, представленным в работе [4] (табл. 1), в структуре сплава были обнаружены округлые включения выделившихся частиц размером $d_{a v}$ в несколько микрон [4], которые образовались в силу ограниченной растворимости $\mathrm{Cr}$ и $\mathrm{Zr}$.

Вклад частиц $\mathrm{Cr}$ в удельное сопротивление (8) (табл. 2) был вычислен с учетом объемной доли частиц вторичной фазы $f_{\sec \mathrm{Cr}}(10)$, зависящей от атомной доли выделившихся атомов $C_{s e c} \mathrm{Cr}(9)$ и числа атомов $\mathrm{Cr}$ в одной частице $N_{p \mathrm{Cr}}$ :

$$
N_{p \mathrm{Cr}}=\frac{\rho_{\text {dens } \mathrm{Cr}} V_{p \mathrm{Cr}}}{\mu_{\mathrm{Cr}} m_{0}}
$$

где $\rho_{\text {dens } \mathrm{Cr}}$ - плотность $\mathrm{Cr} ; \rho_{\text {dens } \mathrm{Cr}}=7.19 \cdot 10^{3} \mathrm{~kg} / \mathrm{m}^{3}$; $\mu_{\mathrm{Cr}}-$ относительная атомная масса $\mathrm{Cr}, \mu_{\mathrm{Cr}}=51.996$; $m_{0}$ - атомная единица массы. Объемная доля $f_{\text {sec } \mathrm{Cr}}(10)$ 
Таблица 2. Модельные значения плотности дислокаций и вкладов дефектов кристаллической структуры в удельное сопротивление сплава $\mathrm{Cu}-1.8 \mathrm{Cr}-0.8 \mathrm{Zr}$

\begin{tabular}{l|c|c}
\hline \multicolumn{1}{c|}{ Состояние } & $\mathrm{SS}$ & 4ЕСАР \\
\hline$\rho_{\text {tot }}, \mathrm{m}^{-2}$ & $1.17 \cdot 10^{11}$ & $7.96 \cdot 10^{14}$ \\
$\rho_{\text {sec } \mathrm{Cr}}, \Omega \cdot \mathrm{m}$ & $6.562 \cdot 10^{-12}$ & $3.506 \cdot 10^{-12}$ \\
$\rho_{\text {sec } \mathrm{Zr}}, \Omega \cdot \mathrm{m}$ & $1.192 \cdot 10^{-11}$ & $6.350 \cdot 10^{-12}$ \\
$\rho_{T}, \Omega \cdot \mathrm{m}$ & $1.741 \cdot 10^{-8}$ & $1.742 \cdot 10^{-8}$ \\
$\rho_{\mathrm{Cr}}, \Omega \cdot \mathrm{m}$ & $2.514 \cdot 10^{-8}$ & $1.978 \cdot 10^{-8}$ \\
$\rho_{\mathrm{Zr}}, \Omega \cdot \mathrm{m}$ & $1.518 \cdot 10^{-8}$ & $1.198 \cdot 10^{-8}$ \\
$\rho_{\text {disl }}, \Omega \cdot \mathrm{m}$ & - & $1.086 \cdot 10^{-11}$ \\
$\rho_{g b}, \Omega \cdot \mathrm{m}$ & $2.108 \cdot 10^{-12}$ & $0.361 \cdot 10^{-8}$ \\
$\rho_{\text {sec } \mathrm{Cr}}^{\prime}, \Omega \cdot \mathrm{m}$ & - & $1.366 \cdot 10^{-9}$ \\
$\rho_{\text {sec } \mathrm{Zr}}^{\prime}, \Omega \cdot \mathrm{m}$ & - & $2.443 \cdot 10^{-9}$ \\
$\rho, 10^{-8} \Omega \cdot \mathrm{m}$ & $5.775 \cdot 10^{-8}$ & $5.662 \cdot 10^{-8}$ \\
$\mathrm{IACS}, \%$ & 29.9 & 30.5 \\
$\mathrm{IACS}, \%$ & $30.0 \pm 0.6$ & $29.0 \pm 0.6$ \\
(эксперимент) & &
\end{tabular}

частиц $\mathrm{Cr}$ при этом имела вид

$$
f_{\text {sec } \mathrm{Cr}}=\frac{W_{\sec \mathrm{Cr}} \alpha}{\rho_{\text {dens } \mathrm{Cr}}\left(\alpha_{1} / \rho_{\text {dens } \mathrm{Cr}}+\alpha_{2} / \rho_{\text {dens } \mathrm{Zr}}+1 / \rho_{\text {dens } \mathrm{Cu}}\right)},
$$

где $\rho_{\text {dens } \mathrm{Cu}}$ - плотность $\mathrm{Cu}, \rho_{\text {dens } \mathrm{Cu}}=8.92 \cdot 10^{3} \mathrm{~kg} / \mathrm{m}^{3}$, $\rho_{\text {dens } \mathrm{Zr}}$ - плотность $\mathrm{Zr}, \rho_{\text {dens } \mathrm{Zr}}=6.506 \cdot 10^{3} \mathrm{~kg} / \mathrm{m}^{3}$

$$
\begin{gathered}
\alpha=n_{\mathrm{Cr}} \frac{\mu_{\mathrm{Cr}}}{\mu_{\mathrm{Cu}}}+n_{\mathrm{Zr}} \frac{\mu_{\mathrm{Zr}}}{\mu_{\mathrm{Cu}}}+1=\alpha_{1}+\alpha_{2}+1, \\
n_{\mathrm{Cr}}=\frac{W_{\mathrm{Cr}}}{1-W_{\mathrm{Cr}}-W_{\mathrm{Zr}}} \frac{\mu_{\mathrm{Cu}}}{\mu_{\mathrm{Cr}}}, \\
n_{\mathrm{Zr}}=\frac{W_{\mathrm{Zr}}}{1-W_{\mathrm{Cr}}-W_{\mathrm{Zr}}} \frac{\mu_{\mathrm{Cu}}}{\mu_{\mathrm{Zr}}} .
\end{gathered}
$$

Здесь $W_{\mathrm{Cr}}, W_{\mathrm{Zr}}$ - массовые доли атомов $\mathrm{Cr}$ и $\mathrm{Zr}$ в сплаве, $W_{\mathrm{Cr}}=1.8 \mathrm{wt} . \%, W_{\mathrm{Zr}}=0.8$ wt.\%. Предполагалось, что объем образца складывается из суммы объемов составляющих его атомов. $\Omega_{\mathrm{Cu}}, \Omega_{\mathrm{Zr}}, \Omega_{\mathrm{Cr}}$ - объемы атомов $\mathrm{Cu}, \mathrm{Zr}$ и $\mathrm{Cr}$ соответственно: $\Omega_{\mathrm{Cu}}=\mu_{\mathrm{Cu}} m_{0} / \rho_{\mathrm{densCu}} \approx$ $\approx 11.82 \cdot 10^{-30} \mathrm{~m}^{3}, \Omega_{\mathrm{Zr}}=\mu_{\mathrm{Zr}} m_{0} / \rho_{\mathrm{densZr}} \approx 23.28 \cdot 10^{-30} \mathrm{~m}^{3}$, $\Omega_{\mathrm{Cr}}=\mu_{\mathrm{Cr}} m_{0} / \rho_{\mathrm{densCr}} \approx 12.00 \cdot 10^{-30} \mathrm{~m}^{3} \cdot \mu_{\mathrm{Zr}}$ - относительная атомная масса $\mathrm{Zr}, \mu_{\mathrm{Zr}}=91.224 ; \mu_{\mathrm{Cu}}$ - относительная атомная масса $\mathrm{Cu}, \mu_{\mathrm{Cu}}=63.5$. Согласно проведенным вычислениям, $f_{\sec \mathrm{Cr}} \approx 1.724 \cdot 10^{-2}$.

Аналогично был вычислен вклад частиц $\mathrm{Cu}_{5} \mathrm{Zr}$ в удельное сопротивление (8) (табл. 2) с учетом объемной доли частиц вторичной фазы $f_{s e c Z r}(10)$, зависящей от атомной доли $C_{s e c Z r}$ выделившихся атомов $\mathrm{Zr}(9)$ и числа атомов $\mathrm{Zr}$ в одной частице $N_{p \mathrm{Zr}}$ :

$$
N_{p \mathrm{Zr}}=\frac{V_{p \mathrm{Zr}}}{5 \Omega_{\mathrm{Cu}}+\Omega_{\mathrm{Zr}}}
$$

Объемная доля $f_{\text {sec } \mathrm{Zr}}$ частиц $\mathrm{Cu}_{5} \mathrm{Zr}$ при этом имела вид

$$
f_{\text {sec } \mathrm{Zr}}=\frac{W_{\text {sec } \mathrm{Zr}} \alpha \beta}{\mu_{\mathrm{Zr}} m_{0}\left(\alpha_{1} / \rho_{\text {dens } \mathrm{Cr}}+\alpha_{2} / \rho_{\text {dens } \mathrm{Zr}}+1 / \rho_{\text {dens } \mathrm{Cu}}\right)}
$$

где $\beta=5 \Omega_{\mathrm{Cu}}+\Omega_{\mathrm{Zr}}$. Ее численное значение оказалось равным $f_{\text {sec Zr }} \approx 3.131 \cdot 10^{-2}$.

Согласно экспериментальным данным, представленным в работе [26], удельное сопротивление чистой электротехнической меди при комнатной температуре равно 1.724-1.8 $\Omega \cdot \mathrm{m}$. Примем вклад в удельное сопротивление сплава тепловых колебаний решетки $\mathrm{Cu}$ равным $\rho_{T \mathrm{Cu}}=1.724 \cdot 10^{-8} \Omega \cdot \mathrm{m}$, что согласуется и с данными работы [9]. Удельное сопротивления $\mathrm{Cr}$ при комнатной температуре равно $\rho_{T \mathrm{Cr}}=2.7 \cdot 10^{-8} \Omega \cdot \mathrm{m}$. Среднее значение удельного сопротивления, обусловленного тепловыми колебаниями решеток $\mathrm{Cu}\left(\rho_{T \mathrm{Cu}}\right), \mathrm{Cr}$ $\left(\rho_{T \mathrm{Cr}}\right)$ и соединения $\mathrm{Cu}_{5} \mathrm{Zr}\left(\rho_{T \mathrm{Zr}}\right)$, определится согласно формуле

$\rho_{T}=\left(1-f_{\sec \mathrm{Cr}}-f_{\sec \mathrm{Zr}}\right) \rho_{T \mathrm{Cu}}+f_{\sec \mathrm{Cr}} \rho_{T \mathrm{Cr}}+f_{\sec \mathrm{Zr}} \rho_{T \mathrm{Zr}}$,

где был принят во внимание вклад в удельное сопротивление областей, занятых частицами $\mathrm{Cr}$ и $\mathrm{Cu}_{5} \mathrm{Zr}$ (табл. 2). Однако при расчетах значение величины $\rho_{T}$ было вычислено, согласно приближенной формуле $\rho_{T}=\left(1-f_{\sec \mathrm{Cr}}\right) \rho_{T \mathrm{Cu}}+f_{\sec \mathrm{Cr}} \rho_{T \mathrm{Cr}}$, без учета вклада частиц $\mathrm{Cu}_{5} \mathrm{Zr}$. Предполагалось, что удельное сопротивление $\rho_{T Z \text { r }}$ соединения $\mathrm{Cu}_{5} \mathrm{Zr}$ имеет порядок удельного сопротивления решетки $\mathrm{Cu}$. При этом вклад частиц $\mathrm{Cu}_{5} \mathrm{Zr}$ в среднее значение $\rho_{T}$ будет на два порядка меньше вклада решетки $\mathrm{Cu}$, так как их объемная доля составляет $f_{\text {sec Zr }} \approx 3.131 \cdot 10^{-2}$.

Вклад в удельное сопротивление растворенных атомов $\mathrm{Cr}$ был оценен, согласно формуле (6) [10] (табл. 2), где атомная доля атомов $\mathrm{Cr}$ равна $C_{s \mathrm{Cr}}=0.488 \mathrm{at} . \%$, $n_{a}=n_{\mathrm{Cu}}-$ объемная концентрация атомов $\mathrm{Cu}$. В случае $\mathrm{Cu} n_{e} \approx n_{\mathrm{Cu}}$. Размеры области взаимодействия электрона с атомом Сr были приняты равными $l_{\mathrm{Cr}}=1.35 b_{\mathrm{B}}[10]$.

Аналогично был вычислен вклад в удельное сопротивление $\rho_{\mathrm{Zr}}$ растворенных атомов $\mathrm{Zr}$ в количестве 0.15 wt.\% (0.104 at.\%) (табл. 2). При этом размеры области взаимодействия электрона с атомом Zr были приняты равными $l_{\mathrm{Zr}}=1.73 b_{\mathrm{B}}$, согласно известному экспериментальному значению удельной электропроводности в SS состоянии сплава (табл. 2). Увеличение размеров области взаимодействия обусловлено наличием разности $Z$ между валентностями $\mathrm{Zr}$ и $\mathrm{Cu}$. Известно, что сечение рассеяния растет как $(\mathrm{Ze})^{2}$, т.е. пропорционально квадрату заряда [27].

Значение средней плотности дислокаций $\rho_{t o t}=f q_{w}+$ $+(1-f) q_{c} \quad$ в исходном состоянии представлено в табл. 2. При этом предполагалось, что дислокации сосредоточены вдоль ГЗ, объемная доля которых была принята равной $f=0.03$ [28]. Они образуют границы зерен. Плотность дислокаций вдоль ГЗ была вычислена согласно формуле $q_{w}=1 /(d \cdot h)$, где $h=h_{c}=7 b_{B}$ при зна- 

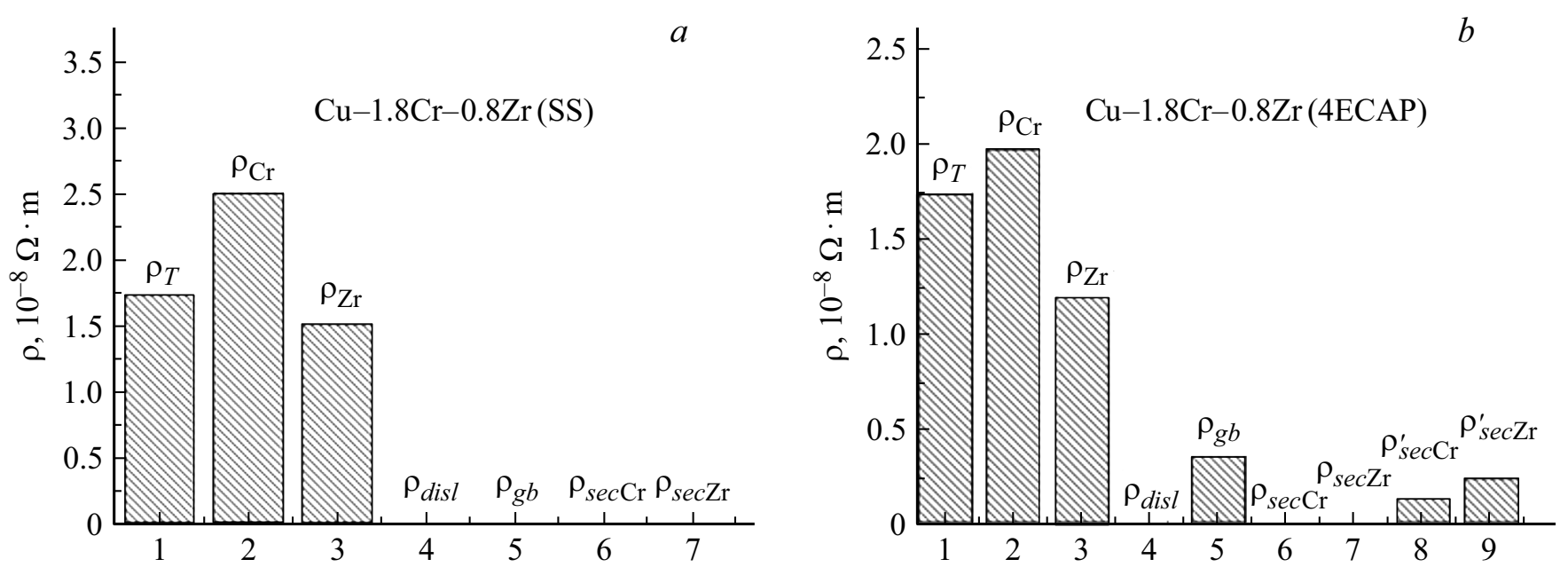

Вклад дефектов кристаллической структуры в удельное сопротивление сплава $\mathrm{Cu}-1.8 \mathrm{Cr}-0.8 \mathrm{Zr}$ в исходном (SS) состоянии $(a)$ и в состоянии после 4 проходов РКУП (4ЕСАР) $(b)$.

чении угла разориентации $\theta=\theta^{*}$, соответствующем границе между областью высоких и малых углов разориентации. В случае сплава $\mathrm{Cu}-1.8$ wt.\% $\mathrm{Cr}-0.8$ wt.\% $\mathrm{Zr}$ плотность дислокаций вдоль ГЗ равна $q_{w}=5.07 \cdot 10^{12} \mathrm{~m}^{-2}$. Плотность дислокаций во внутренних областях зерен равна $q_{c}=0 \mathrm{~m}^{-2}$. Внутренние области зерен были свободны от дислокаций.

В исходном состоянии примерно 78\% ГЗ были высокоугловыми [23]. Среднее значение удельного сопротивления $\rho_{g b}$, обусловленного наличием высокоугловых и $22 \%$ малоугловых $\left(\theta<\theta^{*}\right)$ границ зерен, вычисленное, согласно формулам (5) $\left(\rho_{g b}=0.78\left\langle\rho_{g b}^{h}\right\rangle+0.22\left\langle\rho_{g b}^{l}\right\rangle\right)$, представлено в табл. 2. При этом значение величины $N_{g b}$, вычисленное, согласно микроструктурным данным [4] (табл. 1), было принято равным $N_{g b} \approx 1 / d=9.09 \cdot 10^{3} \mathrm{~m}^{-1}$.

В удельное сопротивление сплава $\mathrm{Cu}-1.8 \mathrm{wt} . \%$ $\mathrm{Cr}-0.8$ wt.\% Zr вносят вклад тепловые колебания кристаллической решетки $\left(\rho_{T}\right)$, частицы вторичной фазы $\left(\rho_{s e c \mathrm{Cr}}, \rho_{\text {sec } \mathrm{Zr}}\right)$, легирующие атомы $\left(\rho_{\mathrm{Cr}}, \rho_{\mathrm{Zr}}\right)$ и границы зерен $\left(\rho_{g b}\right)$. В предположении справедливости правила Маттисена [29], удельное электрическое сопротивление сплава в исходном состоянии было представлено в виде суммы вкладов процессов рассеяния электронов на различных дефектах кристаллической решетки (табл. 2):

$$
\rho=\rho_{T}+\rho_{s e c \mathrm{Cr}}+\rho_{s e c \mathrm{Zr}}+\rho_{\mathrm{Cr}}+\rho_{\mathrm{Zr}}+\rho_{g b} .
$$

Удельное сопротивление сплава в исходном состоянии определяют тепловые колебания атомов в узлах кристаллической решетки и растворенные в матрице легирующие атомы $\mathrm{Cr}$ и $\mathrm{Zr}$. Вклад остальных дефектов кристаллической решетки пренебрежимо мал (см. рисунок, $a)$.

\section{3. Удельное сопротивление сплавов системы $\mathrm{Cu}-\mathrm{Cr}-\mathrm{Zr}$ в состоянии 4ЕCAP}

В процессе РКУП в сплавах сформировалась полосовая УМЗ структура, поперечные размеры элементов которой представлены в табл. 1. Существенно увеличилась плотность дислокаций (табл. 2). В то же время были обнаружены мелкие частицы размером 7-11 nm, которые появились в результате динамического старения при деформировании образцов, что подтверждается характером изменения параметра кристаллической решетки. Предполагалось, что изменение параметра решетки в сплаве $\mathrm{Cu}-1.8$ wt.\% $\mathrm{Cr}-0.8$ wt. $\% \mathrm{Zr}$ в исходном закаленном состоянии и в состоянии после РКУП аналогично таковому в сплаве $\mathrm{Cu}-0.5 \mathrm{wt} . \% \mathrm{Cr}-0.2 \mathrm{wt} . \% \mathrm{Zr}$ в силу одинаковых условий закалки и последующего деформирования. После РКУП параметр решетки уменьшился (табл. 1), что свидетельствует о выделении частиц вторичной фазы $\mathrm{Cr}$ и возможном формировании частиц $\mathrm{Cu}_{5} \mathrm{Zr}$, о чем сообщалось в работе [30].

При изменении параметра решетки Сu на $0.000667 \mathrm{~nm}$ растворилось $0.4 \mathrm{wt} . \% \mathrm{Cr} \quad(0.488 \mathrm{at} \% \mathrm{Cr})$. Изменению решетки на $0.000525 \mathrm{~nm}$ соответствует количество растворенных атомов $\mathrm{Cr}$, равное $W_{s \mathrm{Cr} 2}=0.315 \mathrm{wt} . \%$ $\left(C_{s \mathrm{Cr} 2}=0.384\right.$ at.\%). В то же время доля растворенных атомов $\mathrm{Zr}$ при соответствующем изменении параметра решетки равна $W_{s \mathrm{Zr} 2}=0.118 \mathrm{wt} . \%\left(C_{s \mathrm{Zr} 2}=0.0821\right.$ at.\% $)$. Вклад в удельное сопротивление растворенных атомов $\mathrm{Cr}\left(\rho_{\mathrm{Cr}}\right)$ и $\mathrm{Zr}\left(\rho_{\mathrm{Zr}}\right)$, согласно формуле $(6)$, принимает значения, представленные в табл. 2. При этом оставшаяся часть атомов выделилась в частицы вторичной фазы: $W_{\sec \mathrm{Cr} 2}=0.085$ wt.\% $\left(C_{\text {sec } \mathrm{Cr} 2}=0.104\right.$ at.\% $)$, $W_{\text {sec } \mathrm{Zr} 2}=0.032 \mathrm{wt} . \%\left(C_{\text {sec } \mathrm{Zr} 2}=0.0223\right.$ at.\% $)$.

Частицы $\mathrm{Cr}$ и $\mathrm{Cu}_{5} \mathrm{Zr}$, сформировавшиеся в исходном состоянии, после ИПД стали мельче (табл. 1). Произошло частичное растворение вторичных фаз. 
Согласно формуле (11), число атомов $\mathrm{Cr}$ в одной частице пропорционально ее объему. Число атомов $\mathrm{Cr}$ в крупных частицах в исходном состоянии составляло $N_{p \mathrm{Cr}} \approx 0.766 \cdot 10^{12}$, после РКУП $N_{p \mathrm{Cr} 2} \approx 0.299 \cdot 10^{12}$. Число атомов в частице уменьшилось на $\Delta N_{p \mathrm{Cr}}=0.467 \cdot 10^{12}$.

Массовую долю $W_{s e c \mathrm{Cr}}\left(W_{\text {sec } \mathrm{Cr}}=1.4 \mathrm{wt} . \%\right)$ атомов $\mathrm{Cr}$, выделившихся в частицы вторичной фазы в исходном состоянии, можно представить в виде

$$
\begin{aligned}
& W_{s e c \mathrm{Cr}}=\frac{N_{p \mathrm{Cr} 2} \mu_{\mathrm{Cr}} m_{0}+\Delta N_{p \mathrm{Cr}} \mu_{\mathrm{Cr}} m_{0}}{N_{\mathrm{Cr}} \mu_{\mathrm{Cr}} m_{0}+N_{\mathrm{Zr}} \mu_{\mathrm{Zr}} m_{0}+N_{\mathrm{Cu}} \mu_{\mathrm{Cu}} m_{0}} N_{\rho} \\
& =\frac{N_{p \mathrm{Cr}} N_{p} \mu_{\mathrm{Cr}} m_{0}}{N_{\mathrm{Cr}} \mu_{\mathrm{Cr}} m_{0}+N_{\mathrm{Zr}} \mu_{\mathrm{Zr}} m_{0}+N_{\mathrm{Cu}} \mu_{\mathrm{Cu}} m_{0}}=W_{s e c \mathrm{Cr}}^{\prime}+\Delta W_{s e c \mathrm{Cr}} .
\end{aligned}
$$

Откуда следует, что массовая доля атомов, находящихся в крупных частицах $\mathrm{Cr}$ после РКУП, была равна

$$
W_{s e c \mathrm{Cr}}^{\prime}=W_{s e c \mathrm{Cr}} \frac{N_{p \mathrm{Cr} 2}}{N_{p \mathrm{Cr}}}=0.546 \text { wt.\%(0.667 at.\%). }
$$

Доля атомов $\Delta W_{s e c}$ сr выделилась из крупных частиц в виде мелких частиц $\mathrm{Cr}$, что подтверждается характером изменения параметра решетки. Параметр решетки уменьшился. Следовательно, атомы $\mathrm{Cr}$ могли только выделиться дополнительно из кристаллической решетки. Массовая доля атомов, выделившихся в мелкие частицы $\mathrm{Cr}$, при этом составила

$$
\begin{gathered}
W_{s e c \mathrm{Cr}}^{\prime \prime}=\Delta W_{s e c \mathrm{Cr}}+W_{\sec \mathrm{Cr} 2} \\
\Delta W_{\sec \mathrm{Cr}}=W_{\sec \mathrm{Cr}} \frac{\Delta N_{p \mathrm{Cr}}}{N_{p \mathrm{Cr}}}=0.854 \mathrm{wt} . \% .
\end{gathered}
$$

Согласно проведенным вычислениям, массовая доля атомов $\mathrm{Cr}$, выделившихся в мелкие частицы, составила $W_{s e c \mathrm{Cr}}^{\prime \prime}=0.939 \mathrm{wt} . \%$ (1.146 at.\%). Объемная доля крупных и мелких частиц $\mathrm{Cr}$ (12) была равна соответственно $f_{\text {sec } \mathrm{Cr}}^{\prime} \approx 6.731 \cdot 10^{-3}, f_{\text {sec } \mathrm{Cr}}^{\prime \prime} \approx 1.156 \cdot 10^{-2}$.

Аналогично, согласно формуле (13), число атомов $\mathrm{Zr}$ в частице $\mathrm{Cu}_{5} \mathrm{Zr}$ в исходном состоянии было равно $N_{p \mathrm{Zr}} \approx 0.112 \cdot 10^{12}$. После РКУП число атомов в частице стало равным $N_{p \mathrm{Zr} 2} \approx 0.0436 \cdot 10^{12}$. Число атомов $\mathrm{Zr}$ в частице уменьшилось на $\Delta N_{p Z \mathrm{r}}=0.0684 \cdot 10^{12}$. Массовая доля атомов, находящихся в крупных частицах $\mathrm{Cu}_{5} \mathrm{Zr}$ после РКУП равна

$$
W_{s e c \mathrm{Zr}}^{\prime}=W_{s e c \mathrm{Zr}} \frac{N_{p \mathrm{Zr} 2}}{N_{p \mathrm{Zr}}}=0.253 \text { wt.\%(0.176 at.\%), }
$$

где массовая доля выделившихся в частицы вторичной фазы атомов $\mathrm{Zr}$ была равна $W_{\text {sec } Z \mathrm{r}}=0.65 \mathrm{wt} . \%$. Массовая доля атомов, выделившихся в мелкие частицы, при этом составила

$$
\begin{gathered}
W_{\text {sec } \mathrm{Zr}}^{\prime \prime}=\Delta W_{\text {sec } \mathrm{Zr}}+W_{\text {sec } \mathrm{Zr} 2} \\
\Delta W_{\text {sec } \mathrm{Zr}}=W_{\text {sec } \mathrm{Zr}} \frac{\Delta N_{p \mathrm{Zr}}}{N_{p \mathrm{Zr}}}=0.397 \text { wt.\%. }
\end{gathered}
$$

Согласно проведенным вычислениям, доля атомов $\mathrm{Zr}$, выделившихся в мелкие частицы, составила
$W_{s e c Z r}^{\prime \prime}=0.429$ wt.\% (0.298 at.\%). Объемная доля крупных и мелких частиц $\mathrm{Cu}_{5} \mathrm{Zr}$ (14) после РКУП приняла значения $f_{\text {sec } \mathrm{Zr}}^{\prime} \approx 1.219 \cdot 10^{-2}$ и $f_{\text {sec } \mathrm{Zr}}^{\prime \prime} \approx 2.067 \cdot 10^{-2}$ coответственно,

Полагая средний размер мелких частиц, равным $9 \mathrm{~nm}$, можно оценить вклад частиц $\mathrm{Cr}\left(\rho_{s e c \mathrm{Cr}}^{\prime}\right)$ и частиц $\mathrm{Cu}_{5} \mathrm{Zr}$ $\left(\rho_{s e c Z r}^{\prime}\right)$ в удельное сопротивление УМЗ сплава согласно формуле (8) (табл. 2). Вклад крупных частиц $\mathrm{Cr}\left(\rho_{\sec \mathrm{Cr}}\right)$ и $\mathrm{Cu}_{5} \mathrm{Zr}\left(\rho_{\text {sec }} \mathrm{Zr}\right)$ (табл. 1$)$ в удельное сопротивление УМЗ сплава отражен в табл. 2.

Средняя плотность дислокаций в УМЗ состоянии была вычислена методом, предложенным в работе [31]:

$$
\rho_{t o t}=2 \sqrt{3}\left\langle\varepsilon^{2}\right\rangle^{1 / 2} / D_{X R D} b,
$$

где $D_{X R D}$ - размер областей когерентного рассеяния, $\left\langle\varepsilon^{2}\right\rangle^{1 / 2}$ - величина среднеквадратичных упругих микроискажений кристаллической решетки. Средняя плотность дислокаций была вычислена, согласно формуле (15) (табл. 2), с учетом экспериментальных данных, представленных в табл. 1. Вклад в удельное сопротивление дислокаций во внутренних областях зерен $\rho_{\text {disl }}$ был вычислен с учетом того известного из эксперимента факта, что в ГЗ УМЗ материалов находится $\approx 92 \%$ дислокаций [32] (табл. 2).

Разориентация между зернами в образцах, подвергнутых 4 проходам РКУП, является высокоугловой. Тогда, согласно равенству (5), в УМЗ состоянии сплава вклад ГЗ будет на три порядка больше, чем в его исходном состоянии (табл. 2). При этом значение величины $N_{g b}$, вычисленное согласно микроструктурным данным (табл. 1) [4], было принято равным $N_{g b} \approx 6.45 \cdot 10^{6} \mathrm{~m}^{-1}$.

Среднее значение удельного сопротивления, обусловленного тепловыми колебаниями решеток $\mathrm{Cu}$ и $\mathrm{Cr}$ (табл. 2), было вычислено согласно формуле

$$
\rho_{T}=\left(1-f_{\sec \mathrm{Cr}}^{\prime}-f_{\sec \mathrm{Cr}}^{\prime \prime}\right) \rho_{T \mathrm{Cu}}+\left(f_{\sec \mathrm{Cr}}^{\prime}+f_{\sec \mathrm{Cr}}^{\prime \prime}\right) \rho_{T \mathrm{Cu}} .
$$

При этом, как и в исходном состоянии сплава, вклад частиц $\mathrm{Cu}_{5} \mathrm{Zr}$ в среднее значение величины $\rho_{T}$ не был принят во внимание.

Удельное сопротивление сплава $\mathrm{Cu}-1.8$ wt.\% $\mathrm{Cr}-0.8$ wt.\% $\mathrm{Zr}$ в УМЗ состоянии было вычислено согласно формуле

$$
\begin{aligned}
\rho= & \rho_{T}+\rho_{s e c \mathrm{Cr}}+\rho_{s e c \mathrm{Cr}}^{\prime}+\rho_{s e c \mathrm{Zr}}+\rho_{s e c \mathrm{Zr}}^{\prime} \\
& +\rho_{\mathrm{Cr}}+\rho_{\mathrm{Zr}}+\rho_{d i s l}+\rho_{g b} .
\end{aligned}
$$

Значение удельного сопротивления УМЗ сплава незначительно отличается от такового в исходном состоянии (табл. 2). Оценки, проведенные в настоящей работе, показывают, что увеличение протяженности ГЗ и плотности дислокаций, формирование частиц вторичных фаз не сопровождается значительным увеличением удельного сопротивления УМЗ сплава. Это связано с тем, что увеличение вклада ГЗ и указанных дефектов кристаллической структуры в удельное сопротивление 
сопровождается уменьшением вклада растворенных атомов $\mathrm{Cr}$ и $\mathrm{Zr}$ в результате их выделения в частицы вторичных фаз в процессе динамического старения при РКУП (см. рисунок, $b$ ).

\section{Заключение}

Проведенный анализ удельного сопротивления сплава $\mathrm{Cu}-1.8$ wt.\% $\mathrm{Cr}-0.8$ wt.\% $\mathrm{Zr}$ показал, что в исходном SS состоянии существенный вклад в его удельное сопротивление вносят тепловые колебания атомов в узлах кристаллической решетки и растворенные в $\mathrm{Cu}$ атомы $\mathrm{Cr}$ и Zr. Вклад дислокаций, ГЗ и частиц вторичных фаз оказался пренебрежимо малым. В состоянии 4ЕСАР после 4 проходов РКУП в структуру сплава были дополнительно введены дислокации, увеличилась протяженность ГЗ, выделились мелкие частицы вторичных фаз в результате динамического старения, наблюдалось измельчение имеющихся крупных частиц. Указанные изменения в микроструктуре сплава привели к тому, что вклад растворенных атомов $\mathrm{Cr}$ и $\mathrm{Zr}$ в его удельное сопротивление уменьшился. Однако уменьшение вклада чужеродных атомов компенсируется в значительной степени увеличением вклада ГЗ, мелких частиц $\mathrm{Cr}$ и $\mathrm{Cu}_{5} \mathrm{Zr}$. Вклад дислокаций увеличился на порядок, но его значение на два порядка ниже вклада ГЗ и мелких частиц. Вклад крупных частиц вторичных фаз оказался пренебрежимо малым. Повышением удельного сопротивления ГЗ на 3 порядка по сравнению с таковым в КК состоянии и выделением мелких частиц вторичных фаз объясняется отсутствие выраженного влияния РКУП на электропроводящие свойства сплава. Для понижения его удельного сопротивления при комнатной температуре необходимо после РКУП понизить содержание атомов $\mathrm{Cr}$ и $\mathrm{Zr}$ в результате соответствующей последующей обработки УМЗ образцов.

\section{Конфликт интересов}

Автор заявляет, что у него нет конфликта интересов.

\section{Список литературы}

[1] Islamgaliev R.K., Akhmadeev A., Mulyukov R., Valiev R.Z. // Phys. Stat. Sol. A. 1990. Vol. 118. K27-K29.

[2] Islamgaliev R.K., Murtazin R.Ya., Syutina L.A., Valiev R.Z. // Phys. Stat. Sol. A. 1992. Vol. 129. P. 231-236.

[3] An X.H., Lin Q.Y., Wu S.D., Zhang Z.F., Figueiredo R.B., Gao N., Langdon T.G. // Scripta Mater. 2011. Vol. 64. P. 954957.

[4] Sarkeeva E.A., Sitdikov V.D., Raab G.I., Wei W., Alexandrov I.V. // IOP Conf. Series: Mater. Sci. Engineer. 2018. Vol. 447. P. 012065. DOI: $10.1088 / 1757-899 \mathrm{X} / 447 / 1 / 012065$

[5] Harrison W.A. // J. Phys. Chem. Sol. 1958. Vol. 5. P. 44-46.

[6] Блатт Ф. Физика электронной проводимости в твердых телах. М.: Мир, 1971. 470 с.
[7] Islamgaliev R.K., Nesterov K.M., Bourgon J., Champion Y., Valiev R.Z. // J. Appl. Phys. 2014. Vol. 115. N 19. P. 194301. DOI: $10.1063 / 1.4874655$

[8] Wei K.X., Wei W., Wang F., Du Q.B., Alexandrov I.V., Hu J. // Mater. Sci. Engineer:: A. 2011. Vol. 528. N 3. P. 1478-1484.

[9] Абрикосов А.А. Основы теории металлов. М.: Наука, 1987. $520 \mathrm{c}$.

[10] Chembarisova R.G. IOP Conf. Series: Mater. Sci. Engineer. 2018. Vol. 447. P. 012085. DOI: $10.1088 / 1757-899 X / 447 / 1 / 012085$

[11] Шматов В.T. // ФММ. 1975. Т. 40. № 5. С. 910-919

[12] Glieter H. // Scripta Met. 1977. Vol. 11. P. 305-309.

[13] Каролик А.С. // ФММ. 1993. Т. 75. С. 34-41.

[14] Karolik A.S., Lukhvich A.A. // J. Phys.: Condens. Matter. 1994. Vol. 6. P. 873-886.

[15] Hunter S.C., Nabarro F.R.N. // Proc. Royal Society of London. Series A, Mathematical and Phys. Sci. 1953. Vol. 220. N 1143. P. 542-561. DOI: 10.1098/rspa.1953.0205

[16] Владимиров В.И., Орлов А.Н. // ФТТ. 1969. Т. 11. С. 370 378.

[17] Владимиров В.И., Орлов А.Н. // Проблемы прочности. 1971. № 2. C. 36-38.

[18] Лухвич А.А., Каролик А.С. // ФММ. 1986. Т. 61. Вып. 9. C. 395-398.

[19] Wang Gui-Jin, Vutec V. // Acta Met. 1986. Vol. 34. P. 951960.

[20] Pękala K., Pȩkala M. // NanoStructured Mater. 1995. Vol. 6. P. 819-822.

[21] Чембарисова Р.Г., Александров И.В., Ямилева А.М. // ЖТФ. 2019. Т. 89. Вып. 2. С. 192-201.

DOI: 10.21883/JTF.2019.02.47069.46-18 [Chembarisova R.G., Aleksandrov I.V., Yamileva A.M. // Tech. Phys. 2019. Vol. 64. N 2. P. $162-170$. DOI: $10.1134 / \mathrm{S} 106378421902004 \mathrm{X}$

[22] Осинцев О.Е., Федоров В.Н. Медь и медные сплавы. Отечественные и зарубежные марки. М.: Машиностроение, 2004. $336 \mathrm{c}$.

[23] Шаньгина Д.В. Закономерности получения ультрамелкозернистых медных сплавов с повышенными прочностными и эксплуатационными свойствами. Дисс. на соискание ученой степени канд. техн. наук, ИМЕТ, Москва, (2018).

[24] Николаев А.К., Новиков А.И., Розенберг В.М. Хромовые бронзы. М.: Металлургия, 1983. 175 с.

[25] Диаграммы состояния двойных металлических систем. Справочник. В 3-х т. Т. 2 / Под общ. ред. Н.П. Лякишева. М.: Машиностроение, 1997. 1024 с.

[26] Кузнецов М.И. Основы электротехники. М.: Высшая школа, 1964. $560 \mathrm{c}$.

[27] Займан Джс. Электроны и фононы. М.: ИЛ, 1962. 488 с.

[28] Zehetbauer M.J., Stüwe H.P., Vorhauer A., Schafler E., Kohout J. // Advances engineering materials. 2003. V. 5. N 5. P. 330-337.

[29] Matthiessen A., Vogt C. // Philosophical Transactions - The Royal Society. 1864. Vol. 154. P. 167-200.

[30] Zhang Sh., Li R., Kang H., Chen Z., Wang W., Zou C., Li T., Wang T. // Mater. Sci. Eng. A. 2017. Vol. 680. P. 108-114.

[31] Zhao Y.H, Liao X.Z., Jin Z., Valiev R.Z., Zhu Y.T. Acta Mater 2004. Vol. 52. P. 4589-4599. doi.org/10.1016/j.actamat.2004.06.017

[32] Zaynullina L.I., Sitdikov V.D., Alexandrov I.V., Wei W. IOP Conf. Series: Mater. Sci. Engineer. 2018. Vol. 447. P. 012041. DOI: $10.1088 / 1757-899 X / 447 / 1 / 012041$ 